\title{
Molecular Recognition of Agonist and Antagonist for Peroxisome Proliferator-Activated Receptor- $\alpha$ Studied by Molecular Dynamics Simulations
}

\section{Mengyuan Liu ${ }^{1}$, Lushan Wang ${ }^{2}$, Xian Zhao ${ }^{1, *}$ and Xun Sun ${ }^{1, *}$}

1 State Key Laboratory of Crystal Materials, Shandong University, 27 Shanda Nanlu, Jinan 250100, China; E-Mail: 1myiop@sina.com

2 State Key Laboratory of Microbial Technology, Shandong University, 27 Shanda Nanlu, Jinan 250100, China; E-Mail: 1swang@sdu.edu.cn

* Authors to whom correspondence should be addressed;

E-Mails: zhaoxian@icm.sdu.edu.cn (X.Z.); sunxun@icm.sdu.edu.cn (X.S.);

Tel./Fax: +86-531-8836-6330 (X.Z.).

Received: 19 January 2014; in revised form: 3 May 2014 / Accepted: 5 May 2014 /

Published: 15 May 2014

\begin{abstract}
Peroxisome proliferator activated receptor- $\alpha$ (PPAR- $\alpha)$ is a ligand-activated transcription factor which plays important roles in lipid and glucose metabolism. The aim of this work is to find residues which selectively recognize PPAR- $\alpha$ agonists and antagonists. To achieve this aim, PPAR- $\alpha / 13 \mathrm{M}$ and PPAR- $\alpha / 471$ complexes were subjected to perform molecular dynamics simulations. This research suggests that several key residues only participate in agonist recognition, while some other key residues only contribute to antagonist recognition. It is hoped that such work is useful for medicinal chemists to design novel PPAR- $\alpha$ agonists and antagonists.
\end{abstract}

Keywords: PPAR- $\alpha$; molecular modeling; diabetes

\section{Introduction}

Peroxisome proliferator activated receptors (PPARs) are DNA-binding transcription factors belonging to the nuclear hormone receptor super family [1-4]. To date, three distinct PPAR subtypes have been identified namely PPAR- $\alpha$, PPAR- $\beta$ (also known as PPAR- $\delta$ ) and PPAR- $\gamma$ [5-7]. Among these subtypes, PPAR- $\alpha$ is a key regulator of lipid and glucose metabolism. Activation of PPAR- $\alpha$ can 
increase high density lipoprotein, decrease triglycerides, increase insulin sensitivity and reduce adiposity. Therefore, it becomes an attractive target for treating type II diabetes and its complications [8-12]. Due to this reason, the structures of PPAR- $\alpha$ have been intensively studied at the atomic level in recent years and several X-ray crystal structures of PPAR- $\alpha$ have been determined (Figure 1). The results suggest that the active site of PPAR- $\alpha$ consists of three parts: arm I, arm II and entrance regions [13] (Figure 2).

Figure 1. The overall structures of peroxisome proliferator activated receptor- $\alpha$ (PPAR- $\alpha$ )/ligand complexes. (A) PPAR- $\alpha / 13 \mathrm{M}$ complex; and (B) PPAR- $\alpha / 471$ complex. PPAR- $\alpha$ backbone is shown in ribbon (Helix: white; Strand: yellow; Coil: blue). Agonist and antagonist are shown in sphere (Carbon atom: purple; Oxygen atom: red; Nitrogen atom: blue; Fluorine atom: green).


Based on the obtained crystal structures, lots of researches have been conducted on PPAR- $\alpha$ agonists [14-16]. Besides agonists, the antagonists are also useful because of the need for fully understanding the pharmacology of PPAR- $\alpha$. Thus, new research efforts have been made to explore the potential utility of PPAR- $\alpha$ antagonists [1]. And several PPAR- $\alpha$ antagonists have been reported [17-19].

In order to develop more potent PPAR- $\alpha$ agonists and antagonists, it is necessary to find key residues which only contribute to agonist (or antagonist) recognition. Previous research suggests that agonists form polar interactions with S280, Y314, H440 and Y464, which are responsible for agonist recognition [13]. Besides these residues, other polar residues in the binding pocket can also form strong polar interactions with ligands and participate in agonist (or antagonist) recognition. Considering that apolar interactions are crucial for molecular recognition, we deduce that some hydrophobic residues also play important roles in agonist (or antagonist) recognition. Thus, the aim of this work is to find whether some other residues can be involved in agonist (or antagonist) recognition. So far, the systematic researches on this issue are limited, which may hinder rational design of more potent PPAR- $\alpha$ agonists and antagonists. To achieve this goal, the researchers must determine the interaction strength between ligands and residues in PPAR- $\alpha$, which cannot be compared by only inspecting the crystal structures. Under this condition, molecular dynamics simulation is a useful tool 
to achieve this goal. Thus, conventional molecular dynamics simulations of PPAR- $\alpha$ in complex with an agonist 13M, as well as an antagonist 471 were performed (Figure 3 ). It is hoped that these findings can provide useful information to help medicinal chemists design more potent PPAR- $\alpha$ agonists and antagonists.

Figure 2. The active site of PPAR- $\alpha$. (A) PPAR- $\alpha / 13 \mathrm{M}$ complex; and (B) PPAR- $\alpha / 471$ complex. Residues in PPAR- $\alpha$ are only shown with backbone atoms. Agonist and antagonist are shown in stick with purple carbon atoms. The arm I region is shown in stick with blue atoms. The arm II region is shown in stick with orange atoms. The entrance region is shown in stick with green atoms. For the sake of clarity, only the polar hydrogen atoms are displayed.

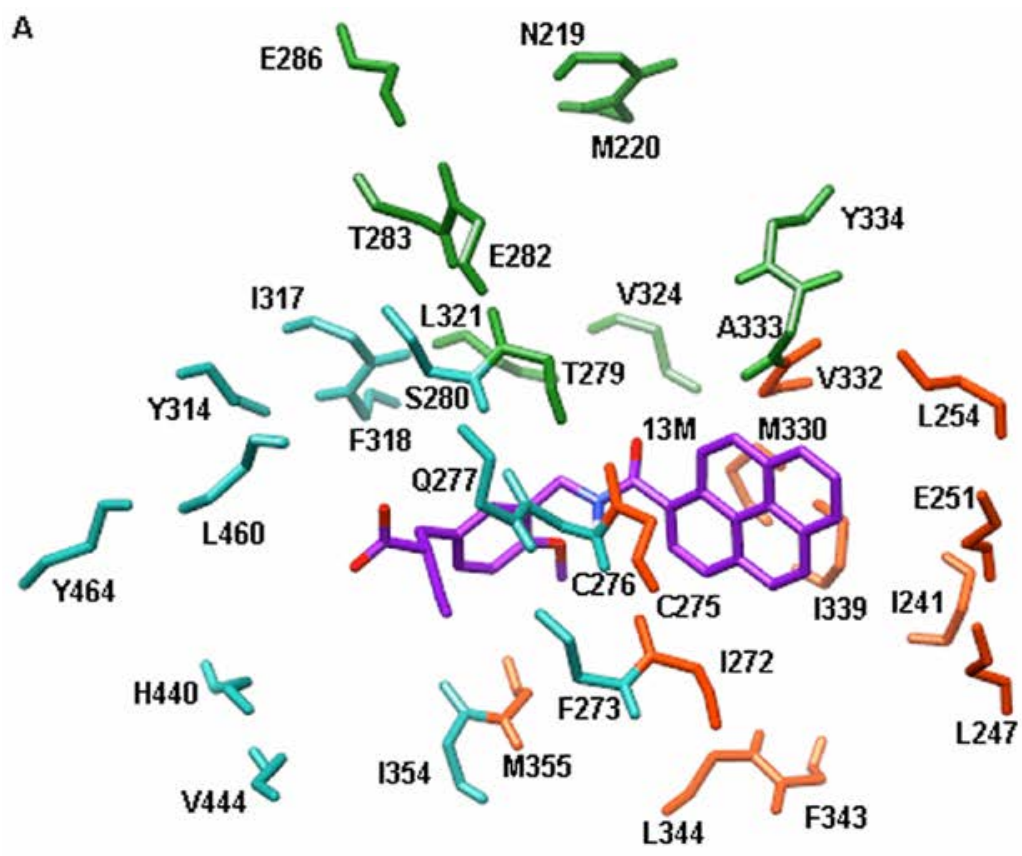

B




Figure 3. Chemical structures of PPAR- $\alpha$ agonist $13 \mathrm{M}$ and antagonist 471.<smiles>CCC(Cc1ccc(OC)c(CNC(=O)c2ccc3ccc4cccc5ccc2c3c45)c1)C(=O)O</smiles><smiles>CCC(=O)NCC(Cc1ccc(OCCc2nc(-c3ccccc3)oc2C)cc1)N/C(C)=C\C(=O)c1ccc(C(F)(F)F)cc1</smiles><smiles>C=[14C][14CH]=[14CH]</smiles>

$$
\begin{gathered}
471 \\
\mathrm{IC}_{50}=240 \mathrm{nM}
\end{gathered}
$$

\section{Results and Discussion}

\subsection{Backbone Stability}

The root mean square deviation (RMSD) for backbone $\mathrm{C} \alpha$ atoms respect to initial structures of production dynamics was calculated. It can be observed form Figure 4 that the RMSD values for PPAR- $\alpha / 13 \mathrm{M}$ complex fluctuate around $0.1 \mathrm{~nm}$ in the period of $20-50 \mathrm{~ns}$, while the values for PPAR- $\alpha / 471$ complex stabilize at about $0.25 \mathrm{~nm}$. These results indicate that both systems reach equilibrium within $20 \mathrm{~ns}$ and the trajectories of the last $30 \mathrm{~ns}$ can be used to perform hydrogen bond and energy decomposition analyses.

Figure 4. The root mean square deviation (RMSD) of $\mathrm{C} \alpha$ atoms for different systems. 13M: PPAR- $\alpha / 13 \mathrm{M}$ complex; 471: PPAR- $\alpha / 471$ complex.

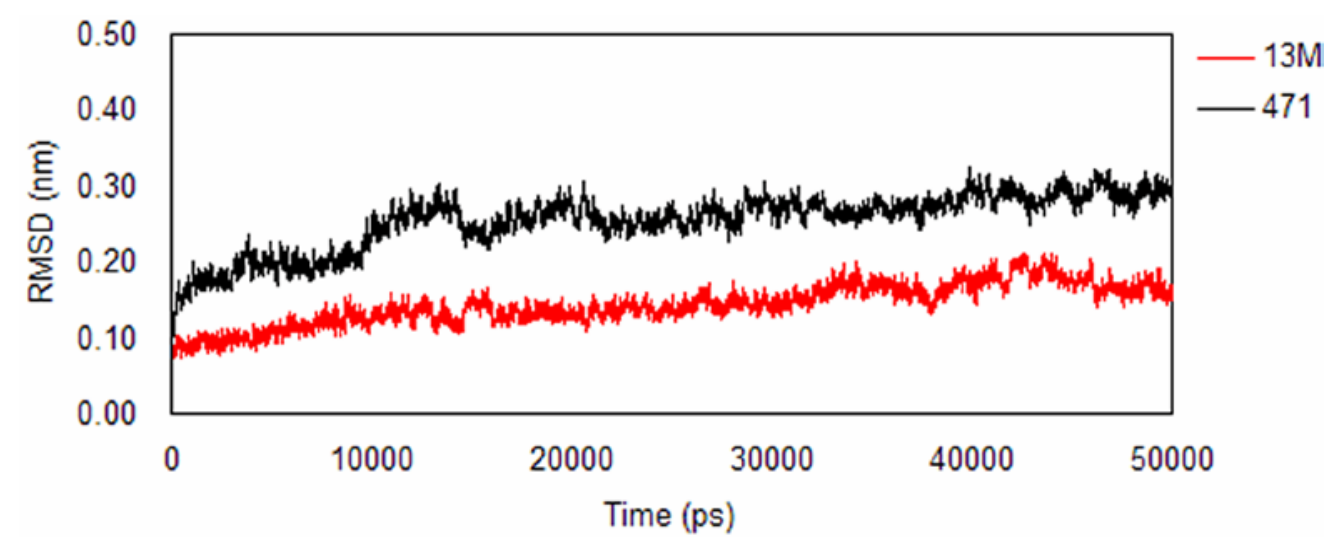

\subsection{Hydrogen Bond Analysis}

Stable hydrogen bonds are crucial for molecular recognition. Residues which form more stable hydrogen bonds with agonist than with antagonist will be considered to only participate in agonist recognition. On the contrary, residues which form more stable hydrogen bonds with antagonist than with agonist will be considered to only participate in antagonist recognition. Considering that the hydrogen bond stability cannot be compared by inspecting the crystal structures, molecular dynamics simulations must be used. Table 1 lists the hydrogen bond probability of ligands with some residues in 
PPAR- $\alpha$. It can be seen that all of the five residues form more stable hydrogen bonds with $13 \mathrm{M}$ than 471, which suggests that Q277, T279, S280, Y314 and H440 only take part in PPAR- $\alpha$ agonist recognition. Among these hydrogen bonds, the hydrogen bonds of $13 \mathrm{M}$ with Q277 and T279 (Figure 5A) cannot be seen from the crystal structure. Based on this finding, we advise medicinal chemists to make designed PPAR- $\alpha$ agonists form hydrogen bonds with Q277 and T279. However, it must be noted that the hydrogen bond stability is not enough to determine which residues can differentiate agonist and antagonist because this analysis only in some extent reflect the electrostatic interactions. To more fully explore the interaction strength of residues with agonists and antagonists, the interaction energies must be calculated.

Table 1. The probability (\%) of hydrogen bonds between PPAR- $\alpha$ and ligands.

\begin{tabular}{ccc}
\hline \multirow{2}{*}{ Residues } & \multicolumn{2}{c}{ Ligands } \\
\cline { 2 - 3 } & $\mathbf{1 3 M}$ & $\mathbf{4 7 1}$ \\
\hline Q277 & 31.1 & 0.0 \\
T279 & 78.5 & 0.1 \\
S280 & 99.7 & 19.1 \\
Y314 & 100.0 & 0.0 \\
H440 & 88.8 & 73.3 \\
\hline
\end{tabular}

Figure 5. Snapshots of PPAR- $\alpha$ /ligand complexes at 50 ns (A) PPAR- $\alpha / 13 \mathrm{M}$ complex; and (B) PPAR- $\alpha / 471$ complex. Agonist and antagonist are shown in stick with purple carbon atoms, while residues of PPAR- $\alpha$ are shown in stick with green carbon atoms. The hydrogen bonds are shown in black lines (For the sake of clarity, only the polar hydrogen atoms are displayed).

A

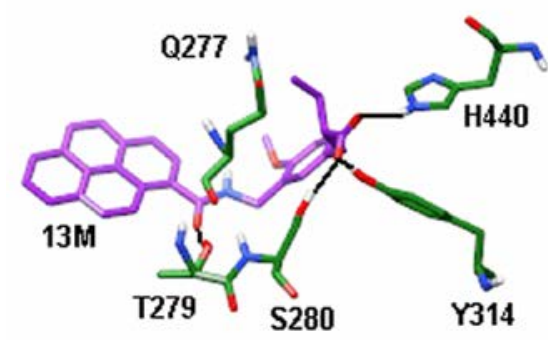

B

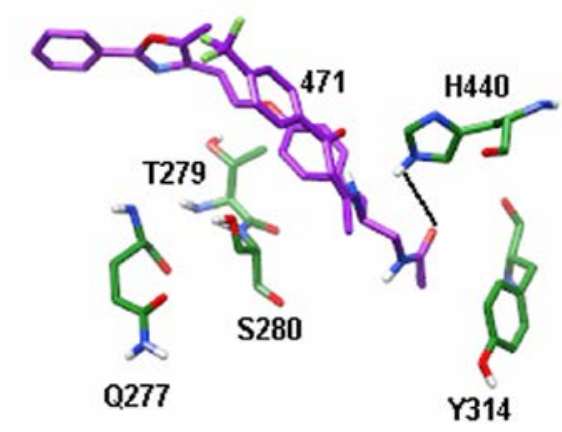

\subsection{Energy Decomposition Analysis}

Considering that both of the $13 \mathrm{M}$ and 471 occupy the arm I, arm II and entrance region, the interaction energies of residues in these regions with $13 \mathrm{M}$ and 471 cannot be judged by inspecting the crystal structures. So the energy decomposition analysis must be carried out. The calculated results are shown in Figure 6. Residues which only exhibit strong interactions with $13 \mathrm{M}$ will be considered to have selectivity for agonist recognition, while residues which only exhibit strong interactions with 471 will be considered to have selectivity for antagonist recognition.

In arm I region, the interaction energies of 13M with Q277, S280, Y314, H440 and Y464 are stronger than 471 (Figure 6A), which indicates that these residues selectively recognize agonists. 
In contrast, 471 binds $\mathrm{I} 317$ and I354 more tightly than 13M (Figure 6A). This suggests that the two residues can selectively recognize antagonists. The previous researches have reported that S280, Y314, H440 and Y464 are responsible for agonist recognition [13], which are consistent with our studies.

In arm II region, the interactions of $13 \mathrm{M}$ with $\mathrm{C} 275$ and V332 are much stronger than 471, indicating that the two residues only make contributions to agonist recognition (Figure 6B). Unlike $\mathrm{C} 275$ and V332, the non-bonded interactions between 471 and 1272 are stronger than 13M, which suggests that this residue can only be responsible for antagonist recognition (Figure 6B).

In entrance region, the difference in interaction energies of ligands with T279 and L321 is significant (Figure 6C). 13M binds these two residues more tight than 471 . Therefore, it can be inferred that T279 and L321 only contribute to agonist recognition.

Figure 6. The average total interaction energies of agonist and antagonist with residues in (A) Arm I region; (B) Arm II region; and (C) Entrance region.

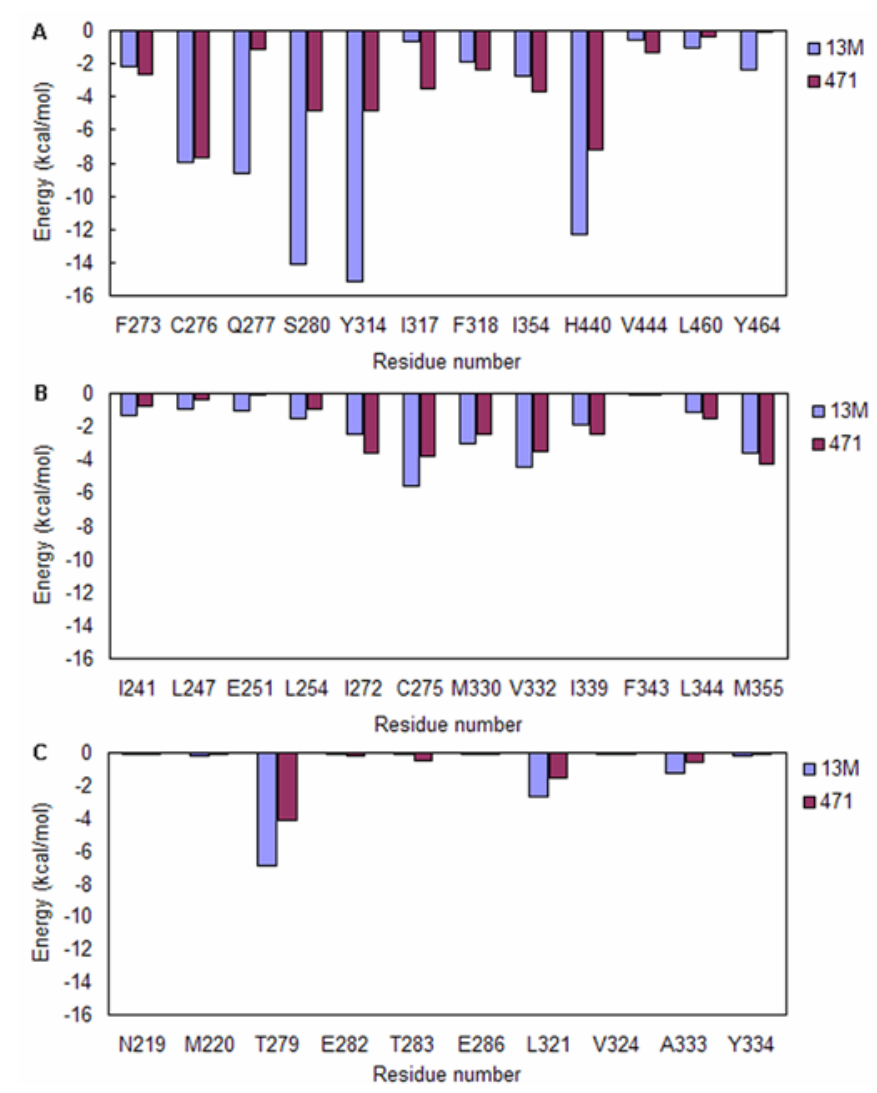

\section{Experimental Section}

\subsection{System Preparation}

The X-ray crystal structures of PPAR- $\alpha / 13 \mathrm{M}$ (PDB code: 3VI8) [20] and PPAR- $\alpha / 471$ (PDB code: 1KKQ) [21] complexes were obtained from the RCSB Protein Data Bank. Crystal water molecules within $4 \AA$ of ligands were kept. For PPAR- $\alpha / 471$ complex, only the A chain was kept. And the co-repressor in PPAR- $\alpha / 471$ complex was also removed because this work is only to explore the interactions of PPAR- $\alpha$ with ligands. Finally, the hydrogen atoms were added by Maestro (Schrodinger LLC, New York, NY, USA). 


\subsection{Molecular Dynamics Simulations}

Molecular dynamics simulations were performed using Gromacs 4.5.3 program [22-25]. The force field for proteins was Amber FF99SB [26,27], while for agonist and antagonist was General Amber Force Field (GAFF) [28]. The systems were immersed in a SPC (simple point charge) water [29] box of $1.0 \mathrm{~nm}$ from the solute surface. The sodium ions were then added to neutralize the systems. The Particle Mesh Ewald (PME) method [30-32] was used for correcting electrostatic interaction. The LINCS algorithm [33,34] was employed to constrain all bonds involving hydrogen atoms. Periodic boundary conditions were also used. The non-bonded cutoff distance was set to $1.0 \mathrm{~nm}$. The temperature was kept at $300 \mathrm{~K}$ with V-rescale temperature coupling [35]. The time step was $1.0 \mathrm{fs}$. The trajectories were sampled every $10 \mathrm{ps}$ in molecular dynamics simulations. Steepest descent energy minimization was first performed to give the maximum force below $1000 \mathrm{~kJ} \cdot \mathrm{mol}^{-1} \cdot \mathrm{nm}^{-2}$ in order to remove the steric clash. After that, the complexes were then equilibrated by 100 ps position restraint MD simulations with $1000 \mathrm{~kJ} \cdot \mathrm{mol}^{-1} \cdot \mathrm{nm}^{-2}$ constant force on the heavy atoms of proteins and ligands under NVT (constant number of molecules, volume and temperature) condition. The X-ray crystal structure of PPAR- $\alpha / 13 \mathrm{M}$ complex missed residues 231-237 and 263-264. But these residues are far away from the binding sites of agonist $13 \mathrm{M}$. Therefore, the impact of the missing residues on agonist binding is limited. Considering this, $1 \mathrm{~ns}$ equilibrium simulation and $50 \mathrm{~ns}$ production run with restraints on the $\mathrm{C} \alpha$ atoms of residues 230, 238, 262 and 265 (restraint force constant $=1000 \mathrm{~kJ} \cdot \mathrm{mol}^{-1} \cdot \mathrm{nm}^{-2}$ ) were sequentially carried out under NVT condition. Unlike PPAR- $\alpha / 13 \mathrm{M}$ complex, no missing residue was found in PPAR- $\alpha / 471$ complex. So no position restraints were applied in the above $1 \mathrm{~ns}$ equilibrium simulation and $50 \mathrm{~ns}$ production run under NVT condition.

\subsection{Hydrogen Bond Analysis}

To define the presence of hydrogen bond, an acceptor-donor distance within $0.35 \mathrm{~nm}$, and an acceptor-hydrogen-donor angle within $30^{\circ}$ was used [36]. The probability of hydrogen bond was calculated using the following equation [37]:

$$
P_{\text {hbond }}=\frac{N_{\text {existence }}}{N_{\text {total }}} \times 100 \%
$$

where $P_{\text {hbond }}$ was the probability of hydrogen bond. $N_{\text {existence was the number of frames that }}$ investigated hydrogen bonds existed. $N_{\text {total }}$ was the total number of collected frames in production phase. The probability of each hydrogen bond was calculated in terms of a percentage that varied from $0 \%$ to $100 \%$, where a percentage of 100 indicated that the hydrogen bond was highly stable and a percentage of 0 indicated an unstable hydrogen bond. Three thousand snapshots isolated from the last $30 \mathrm{~ns}$ production runs with an interval of $10 \mathrm{ps}$ were employed for hydrogen bond analysis.

\subsection{Energy Decomposition Analysis}

The electrostatic $\left(E_{\text {elec }}\right)$ and van der Waals $\left(E_{\mathrm{vdw}}\right)$ interaction energies of some residues in PPAR- $\alpha$ with ligands were calculated according to the Amber force field equation. The total interaction energies between residues in PPAR- $\alpha$ with ligands are the sum of $E_{\text {elec }}$ and $E_{\mathrm{vdw}}$. All energy components are calculated using the same snapshots as the hydrogen bond analysis. 


\section{Conclusions}

In conclusion, the hydrogen bond and energy decomposition analyses suggest that S280, Y314, H440 and Y464 only participate in agonist recognition, which is accord with the previous reports [13]. What is more, our research suggests that C275, Q277, T279, L321 and V332 are only involved in agonist recognition, while I272, I317 and I354 only contribute to antagonist recognition. It is advised that medicinal chemists can make strong non-bonded interactions (such as hydrogen bonds, salt bridges and $\pi-\pi$ stacking interactions) with the above residues when they design PPAR- $\alpha$ agonists and antagonists.

\section{Author Contributions}

Mengyuan Liu wrote the manuscript and carried out molecular dynamics simulations. Lushan Wang, Xian Zhao and Xun Sun designed the experiments and revised manuscript.

\section{Conflicts of Interest}

The authors declare no conflict of interest.

\section{References}

1. Ammazzalorso, A.; Giancristofaro, A.; D’Angelo, A.; Filippis, B.D.; Fantacuzzi, M.; Giampietro, L.; Maccallini, C.; Amoroso, R. Benzothiazole-based $N$-(phenylsulfonyl)amides as a novel family of PPAR $\alpha$ antagonists. Bioorg. Med. Chem. Lett. 2011, 21, 4869-4872.

2. Dhoke, G.V.; Gangwal, R.P.; Sangamwar, A.T. A combined ligand and structure based approach to design potent PPAR- $\alpha$ agonists. J. Mol. Struct. 2012, 1028, 22-30.

3. Ricote, M.; Huang, J.T.; Welch, J.S.; Glass, C.K. The peroxisome proliferator-activated receptor $(\operatorname{PPAR} \gamma)$ as a regulator of monocyte/macrophage function. J. Leukoc. Biol. 1999, 66, 733-739.

4. Jiang, X.; Lee, G.T.; Prasad, K.; Repič, O. A practical synthesis of a diazepinylbenzoic acid, a retinoid X receptor antagonist. Org. Process Res. Dev. 2008, 12, 1137-1141.

5. Pingali, H.; Jain, M.; Shah, S.; Makadia, P.; Zaware, P.; Goel, A.; Patel, M.; Giri, S.; Patel, H.; Patel, P. Design and synthesis of novel oxazole containing 1,3-dioxane-2-carboxylic acid derivatives as PPAR $\alpha / \gamma$ dual agonists. Bioorg. Med. Chem. 2008, 16, 7117-7127.

6. Tsao, T.; Kornblau, S.; Safe, S.; Watt, J.C.; Ruvolo, V.; Chen, W.; Qiu, Y.; Coombes, K.R.; $\mathrm{Ju}, \mathrm{Z}$; Abdelrahim, M.; et al. Role of peroxisome proliferator-activated receptor- $\gamma$ and its coactivator DRIP205 in cellular responses to CDDO (RTA-401) in acute myelogenous leukemia. Cancer Res. 2010, 70, 4949-4960.

7. Julan, L.; Guan, H.; van Beek, J.P.; Yang, K. Peroxisome proliferator-activated receptor $\delta$ suppresses 11ß-hydroxysteroid dehydrogenase type 2 gene expression in human placental trophoblast cells. Endocrinology 2005, 146, 1482-1490.

8. Liu, X.-Y.; Wang, R.-L.; Xu, W.-R.; Tang, L.-D.; Wang, S.-Q.; Chou, K.-C. Docking and molecular dynamics simulations of peroxisome proliferator activated receptors interacting with pan agonist sodelglitazar. Protein Pept. Lett. 2011, 18, 1021-1027. 
9. Han, H.O.; Kim, S.H.; Kim, K.-H.; Hur, G.-C.; Yim, H.J.; Chung, H.-K.; Woo, S.H.; Koo, K.D.; Lee, C.-S.; Koh, S.-J.; et al. Design and synthesis of oxime ethers of $\alpha$-acyl- $\beta$-phenylpropanoic acids as PPAR dual agonists. Bioorg. Med. Chem. Lett. 2007, 17, 937-941.

10. Zhang, H.; Ryono, D.E.; Devasthale, P.; Wang, W.; O’Malley, K.; Farrelly, D.; Gu, L.; Harrity, T.; Cap, M.; Chu, C.; et al. Design, synthesis and structure-activity relationships of azole acids as novel, potent dual PPARa/ $\gamma$ agonists. Bioorg. Med. Chem. Lett. 2009, 19, 1451-1456.

11. Ichihara, S.; Obata, K.; Yamada, Y.; Nagata, K.; Noda, A.; Ichihara, G.; Yamada, A.; Kato, T.; Izawa, H.; Murohara, T.; et al. Attenuation of cardiac dysfunction by a PPAR- $\alpha$ agonist is associated with down-regulation of redox-regulated transcription factors. J. Mol. Cell. Cardiol. 2006, 41, 318-329.

12. Guerre-Millo, M.; Gervois, P.; Raspé, E.; Madsen, L.; Poulain, P.; Derudas, B.; Herbert, J.-M.; Winegar, D.A.; Willson, T.M.; Fruchart, J.-C.; et al. Peroxisome proliferator-activated receptor $\alpha$ activators improve insulin sensitivity and reduce adiposity. J. Biol. Chem. 2000, 275, 16638-16642.

13. Zoete, V.; Grosdidier, A.; Michielin, O. Peroxisome proliferator-activated receptor structures: Ligand specificity, molecular switch and interactions with regulators. Biochim. Biophys. Acta 2007, 1771, 915-925.

14. Cronet, P.; Petersen, J.F.W.; Folmer, R.; Blomberg, N.; Sjöblom, K.; Karlsson, U.; Lindstedt, E.-L.; Bamberg, K. Structure of the PPAR $\alpha$ and $\gamma$ ligand binding domain in complex with AZ 242; ligand selectivity and agonist activation in the PPAR family. Structure 2001, 9, 699-706.

15. Li, J.; Kennedy, L.J.; Shi, Y.; Tao, S.; Ye, X.-Y.; Chen, S.Y.; Wang, Y.; Hernández, A.S.; Wang, W.; Devasthale, P.V.; et al. Discovery of an oxybenzylglycine based peroxisome proliferator activated receptor $\alpha$ selective agonist 2-((3-((2-(4-chlorophenyl)-5-methyloxazol-4-yl)methoxy)benzyl) (methoxycarbonyl)am ino)acetic acid (BMS-687453). J. Med. Chem. 2010, 53, 2854-2864.

16. Xu, H.E.; Lambert, M.H.; Montana, V.G.; Plunket, K.D.; Moore, L.B.; Collins, J.L.; Oplinger, J.A.; Kliewer, S.A.; Gampe, R.T.; McKee, D.D.; et al. Structural determinants of ligand binding selectivity between the peroxisome proliferator-activated receptors. Proc. Natl. Acad. Sci. USA 2001, 98, 13919-13924.

17. Sagar, D.R.; Kendall, D.A.; Chapman, V. Inhibition of fatty acid amide hydrolase produces PPAR- $\alpha$-mediated analgesia in a rat model of inflammatory pain. Br. J. Pharmacol. 2008, 155, 1297-1306.

18. Cwinn, M.A.; Jones, S.P.; Kennedy, S.W. Exposure to perfluorooctane sulfonate or fenofibrate causes PPAR- $\alpha$ dependent transcriptional responses in chicken embryo hepatocytes. Comp. Biochem. Phys. C 2008, 148, 165-171.

19. Donnelly, L.E.; Newton, R.; Kennedy, G.E.; Fenwick, P.S.; Leung, R.H.; Ito, K.; Russell, R.E.; Barnes, P.J. Anti-inflammatory effects of resveratrol in lung epithelial cells: Molecular mechanisms. Am. J. Physiol.-Lung C 2004, 287, L774-L783.

20. Kuwabara, N.; Oyama, T.; Tomioka, D.; Ohashi, M.; Yanagisawa, J.; Shimizu, T.; Miyachi, H. Peroxisome proliferator-activated receptors (PPARs) have multiple binding points that accommodate ligands in various conformations: phenylpropanoic acid-type PPAR ligands bind to PPAR in different conformations, depending on the subtype. J. Med. Chem. 2012, 55, 893-902.

21. Xu, H.E.; Stanley, T.B.; Montana, V.G.; Lambert, M.H.; Shearer, B.G.; Cobb, J.E.; McKee, D.D.; Galardi, C.M.; Plunket, K.D.; Nolte, R.T.; et al. Structural basis for antagonist-mediated recruitment of nuclear co-repressors by PPAR $\alpha$. Nature 2002, 415, 813-817. 
22. Hess, B.; Kutzner, C.; van der Spoel, D.; Lindahl, E. GROMACS 4: Algorithms for highly efficient, load-balanced, and scalable molecular simulation. J. Chem. Theory Comput. 2008, 4, 435-447.

23. Berendsen, H.J.C.; van der Spoel, D.; van Drunen, R. GROMACS: A message-passing parallel molecular dynamics implementation. Comput. Phys. Commun. 1995, 91, 43-56.

24. Pronk, S.; Páll, S.; Schulz, R.; Larsson, P.; Bjelkmar, P.; Apostolov, R.; Shirts, M.R.; Smith, J.C.; Kasson, P.M.; van der Spoel, D.; et al. GROMACS 4.5: A high-throughput and highly parallel open source molecular simulation toolkit. Bioinformatics 2013, 29, 845-854.

25. Van der Spoel, D.; Lindahl, E.; Hess, B.; Groenhof, G.; Mark, A.E.; Berendsen, H.J.C. GROMACS: Fast, flexible, and free. J. Comput. Chem. 2005, 26, 1701-1718.

26. Lindorff-Larsen, K.; Piana, S.; Palmo, K.; Maragakis, P.; Klepeis, J.L.; Dror, R.O.; Shaw, D.E. Improved side-chain torsion potentials for the Amber ff99SB protein force field. Proteins 2010, $78,1950-1958$.

27. Hornak, V.; Abel, R.; Okur, A.; Strockbine, B.; Roitberg, A.; Simmerling, C. Comparison of multiple Amber force fields and development of improved protein backbone parameters. Proteins 2006, 65, 712-725.

28. Wang, J.; Wolf, R.M.; Caldwell, J.W.; Kollman, P.A.; Case, D.A. Development and testing of a general amber force field. J. Comput. Chem. 2004, 25, 1157-1174.

29. Zielkiewicz, J. Structural properties of water: Comparison of the SPC, SPCE, TIP4P, and TIP5P models of water. J. Chem. Phys. 2005, 123, 104501.

30. Abraham, M.J.; Gready, J.E. Optimization of parameters for molecular dynamics simulation using smooth particle-mesh Ewald in GROMACS 4.5. J. Comput. Chem. 2011, 32, 2031-2040.

31. Essmann, U.; Perera, L.; Berkowitz, M.L.; Darden, T.; Lee, H.; Pedersen, L.G. A smooth particle mesh Ewald method. J. Chem. Phys. 1995, 103, 8577-8593.

32. Darden, T.; York, D.; Pedersen, L. Particle mesh Ewald: An $N \log (N)$ method for Ewald sums in large systems. J. Chem. Phys. 1993, 98, 10089-10092.

33. Hess, B.; Bekker, H.; Berendsen, H.J.C.; Fraaije, J.G.E.M. LINCS: A linear constraint solver for molecular simulations. J. Comput. Chem. 1997, 18, 1463-1472.

34. Hess, B. P-LINCS: A parallel linear constraint solver for molecular simulation. J. Chem. Theory Comput. 2008, 4, 116-122.

35. Bussi, G.; Donadio, D.; Parrinello, M. Canonical sampling through velocity rescaling. J. Chem. Phys. 2007, 126, 014101.

36. Cosentino, U.; Pitea, D.; Moro, G.; Saracino, G.A.A.; Villa, A. Conformational behaviour determines the low-relaxivity state of a conditional MRI contrast agent. Phys. Chem. Chem. Phys. 2009, 11, 3943-3950.

37. Wang, Q.; Gao, J.; Liu, Y.; Liu, C. Molecular dynamics simulation of the interaction between protein tyrosine phosphatase 1B and aryl diketoacid derivatives. J. Mol. Graph. Model. 2012, 38, 186-193.

(C) 2014 by the authors; licensee MDPI, Basel, Switzerland. This article is an open access article distributed under the terms and conditions of the Creative Commons Attribution license (http://creativecommons.org/licenses/by/3.0/). 